\title{
Building a Foundation for a Digital Maps Collection
}

\author{
Elizabeth Skene \\ Western Carolina University \\ emskene@email.wcu.edu
}

Krista Schmidt

Western Carolina University

kschmidt@email.wcu.edu

Western North Carolina has many treasures, including some buried underwater. Among these subaquatic resources are the vestiges of former communities, hidden by acres of mountain lakes. Homesteads, bridges, roads, churches, schools, and other physical remnants of these communities disappeared as dams were built across the region for both hydroelectric and recreational purposes. One such lake is Lake Lure in Rutherford County, North Carolina, created primarily for recreational uses in
1925-1926. An inquiry from representatives of the town of Lake Lure spurred our academic library's foray into developing a digital collection of historic and regional maps. Town representatives wondered whether Hunter Library, serving Western Carolina University in the Appalachian Mountains, had an interest in historic maps of the Lake Lure area before dam construction. The authors of this paper, the Maps Librarian and the Special Collections Librarian at the institution, took on this project.

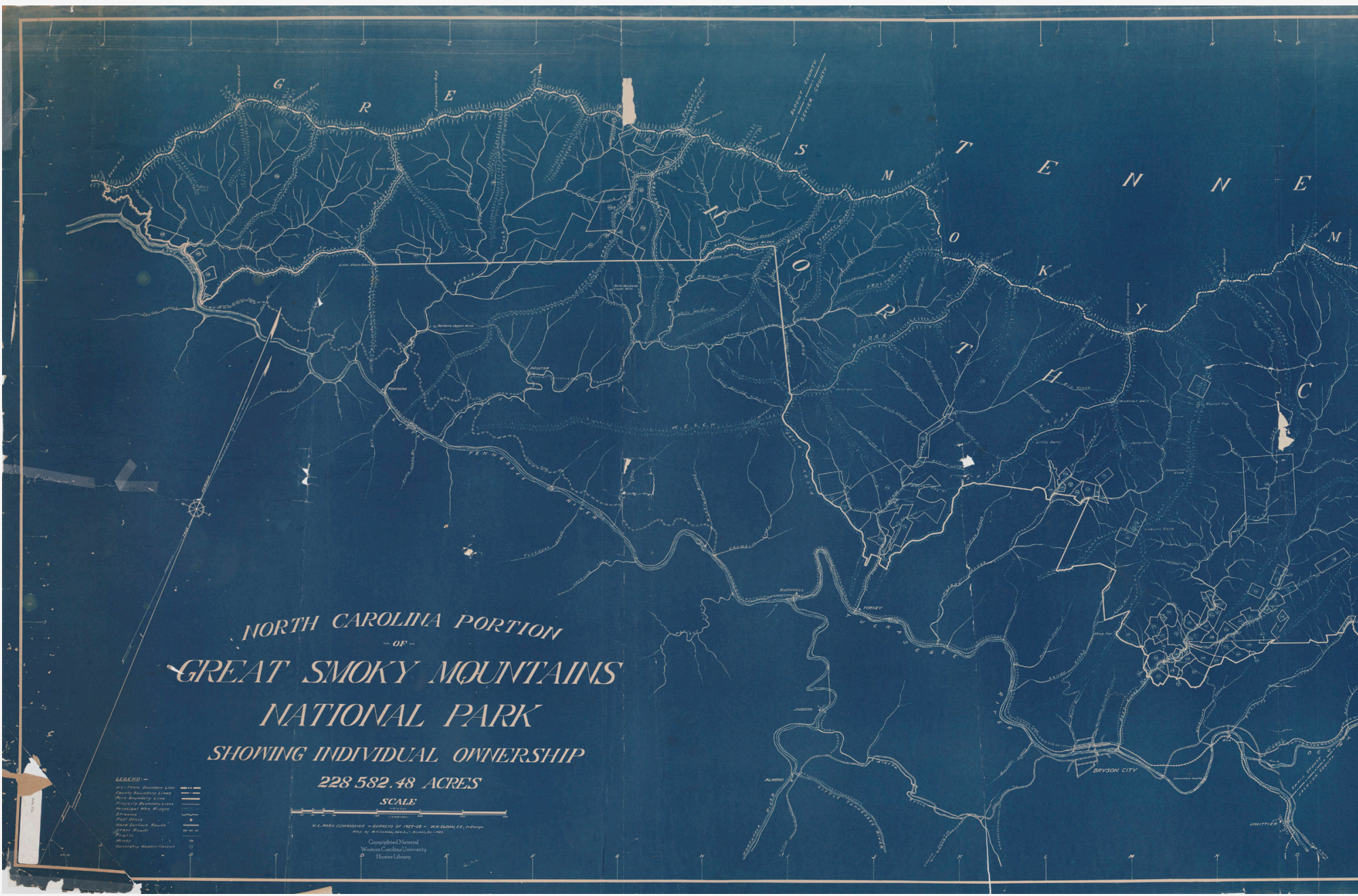

Figure 1. North Carolina Portion of Great Smoky Mountains National Park, Combs and Sloan, North Carolina Park Commission, 1928. 
The majority of these maps, beautifully rendered by Earle Sumner Draper in the early part of the twentieth century, outlined a planned community-complete with subdivided lots-meant to be marketed to potential summer residents from across the Eastern Seaboard. The community was never realized, however, and the maps became a historic record. We were keenly interested in these maps and suggested digitizing them. When our potential partners pressed for details and examples, however, we lacked a comparable digital collection to demonstrate how the digitization would be realized. Creating a pilot digital project for area maps seemed the best option, as this approach would enable us to encounter and manage issues internally before engaging with external partners. We highly value local, unique content and recognized that maps have special value for many reasons. These include documenting place names, family names, and changes to landscapes over time. We hoped that this pilot would allow us to build partnerships and preserve materials throughout the region.
We began the pilot project by defining the collection's scope. We selected and prioritized maps in our collection that were of local interest or of regional importance, allowing us to create a framework for inclusion that was reasonable in scale for a pilot project. We first determined that the digital collection would be limited to Western North Carolina, which is made up of the twenty-four westernmost counties of the state. Due to its proximity, we also chose to include the Great Smoky Mountains National Park, which straddles both North Carolina and Tennessee. Those limitations still included a surprising number of maps, so we further narrowed our efforts to maps unique in subject and likely to be either the only copy in existence or one of a very few copies. The final framework gave us a diverse collection from which to draw, one that was interesting yet not overwhelming in number. The final pilot collection included old plans of our university, orthophoto maps illustrating development around the university and local area, hand-drawn maps relating to the area's Eastern

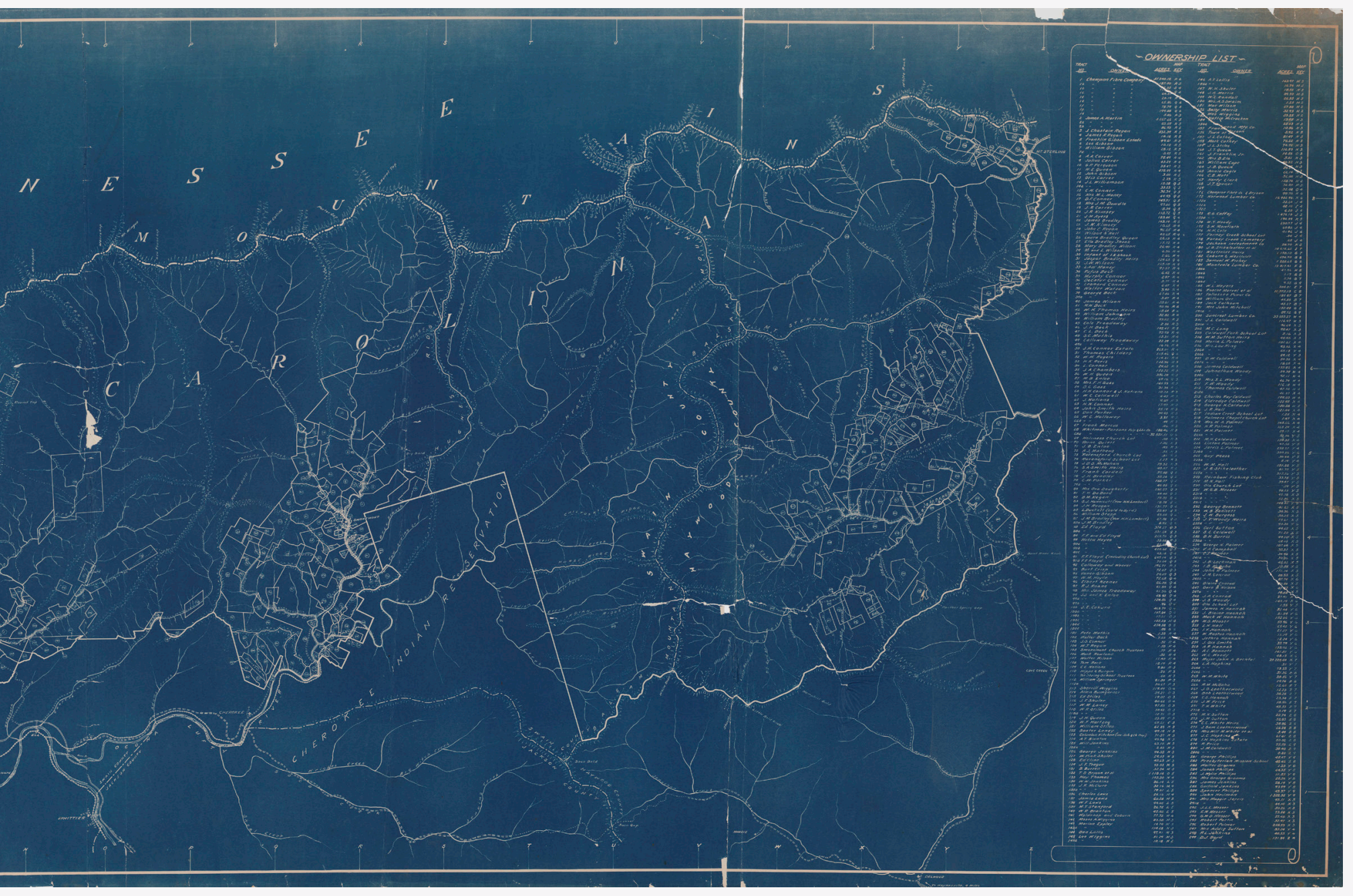

Figure 1 (continued). 
Band of Cherokee Indians, and maps indicating historic family holdings or defunct place names.

The next step was to determine how to make the maps as accessible as possible. We knew we wanted to include each map in both the library's traditional catalog (part of our integrated library system [ILS]), as well as the digital collections platform, CONTENTdm. Each platform offered unique strengths. For example, including an ILS catalog record for the maps provided more visibility, as the library catalog is often the first point of contact for patrons doing research. Information in the catalog record included the title, publisher, date, subject headings, and a link to the map in the digital collection. The digital collection's strengths included a high-resolution version of each image that users can scroll, zoom, or view in full screen. It also allowed us to perform optical character recognition on each record, meaning that, in addition to any information provided in the description of the item, any printed text on the map itself was searchable. Therefore, information about the map not captured in the official description was still findable and provided an additional access point.

Since maps would be made available through both platforms, it was important that descriptive information created by the library's cataloging unit accommodated the different requirements of each. This descriptive information, called metadata, was outlined in a schema that detailed how maps should be described in both the ILS and in CONTENTdm. The document detailed each field that patrons would see when viewing a map in the digital collection. This schema also ensured that the descriptions adhered to library cataloging best practices, as well as the two most widely used descriptive standards in libraries, MARC and Dublin Core. For example, the field name that displays as "Date Depicted" within the digital collections correlates to MARC field 050 and Dublin Core field "Coverage-Temporal." Finally, we also decided which fields would be hidden or searchable within the digital collections.

Considerable thought was given to supplemental metadata that provided information about what the map depicted rather than about the map itself. To increase findability, we included a list of places depicted in each map if that information was not already provided in the description. For example, if a map of Jackson County included symbols indicating the presence of mines, we might add the

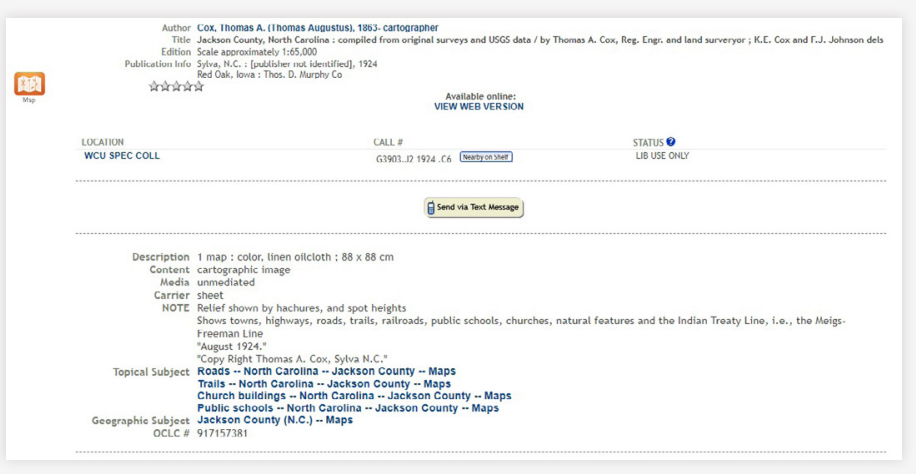

Figure 2a. ILS record.

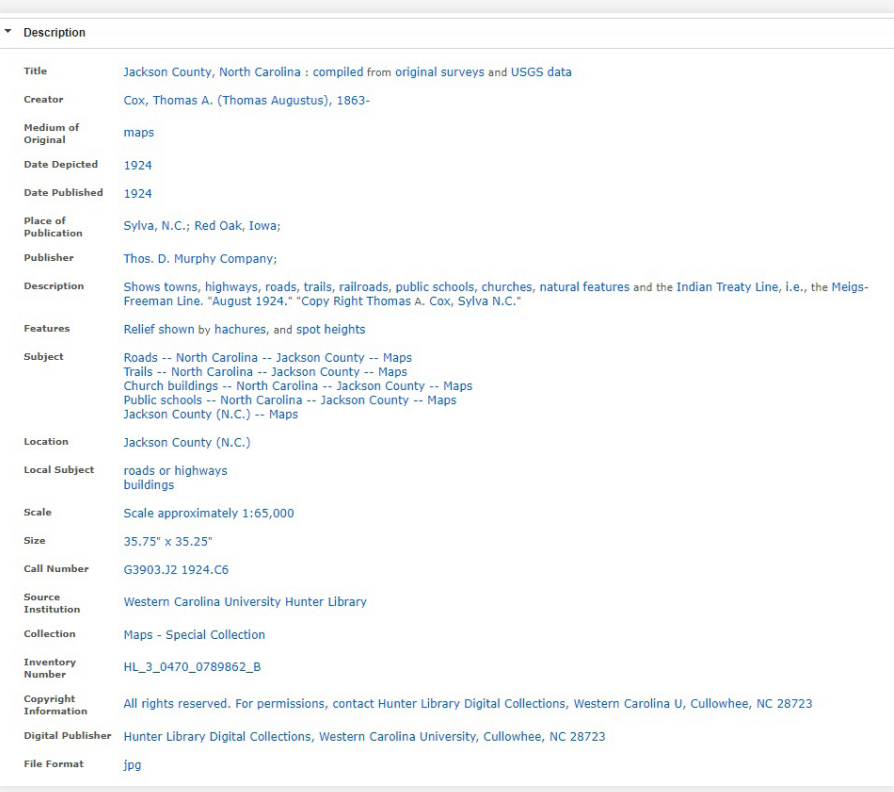

Figure $2 \mathrm{~b}$. CONTENTdm record.

\begin{tabular}{|c|c|c|c|c|c|}
\hline \multicolumn{6}{|c|}{$\begin{array}{l}\text { Western North Carolina Regional Map Collection } \\
\text { (Example info based on map record b59051590) }\end{array}$} \\
\hline $\begin{array}{l}\text { Field Names } \\
\text { In } \\
\text { CONTENTdm }\end{array}$ & $\begin{array}{c}\text { MARC } \\
\text { Tags }\end{array}$ & $\begin{array}{l}\text { Dublin Core } \\
\text { Map }\end{array}$ & Hide & $\begin{array}{l}\text { Field } \\
\text { Values }\end{array}$ & Example \\
\hline Staff Notes & & None & Yes & & \\
\hline Title & $245 \$ a \$ b$ & Title & & & $\begin{array}{l}\text { General Plan for development of } \\
\text { campus of Western Carolina } \\
\text { Teachers College, Cullowhee, N.C. } \\
\text { Not } \$ c \text { - nothing after / }\end{array}$ \\
\hline Creator & $1 \times x$ & Creator & & Controlled & Draper, E. S.; do not include Se \\
\hline Contributor & $7 \times x$ & Contributor & & Controlled & \\
\hline $\begin{array}{c}\text { Medium of } \\
\text { Original } \\
\end{array}$ & & Format-Medium & & $\begin{array}{c}\text { Controlled- } \\
\text { shared }\end{array}$ & Maps \\
\hline Type & & Type & Yes & $\begin{array}{c}\text { Controlled- } \\
\text { shared }\end{array}$ & Still Image \\
\hline Date Depicted & $\begin{array}{c}050 \text { (take from } \\
\text { call } \\
\text { no./situation } \\
\text { no.) }\end{array}$ & $\begin{array}{l}\text { Coverage- } \\
\text { Temporal }\end{array}$ & & & 1927 \\
\hline
\end{tabular}

Figure 3. Metadata schema. 
word "mines" to the description, even if the map's focus was not mining. We also developed a list of terms to provide a more intuitive way of browsing than official Library of Congress Subject Headings. These terms are specific to our institution, location, and interests, and are considered locally authoritative rather than broadly applicable to other libraries. Examples used were "advertisements," "cemeteries," "mountain range," and "tourist maps."
To include the maps in the library's ILS and to provide high quality descriptive information for each, a part-time cataloger dedicated to the project began working on the selected maps. After an initial meeting to determine the metadata schema, the cataloger and the Maps Librarian discussed other cataloging practices unique to the collection. This discussion included determining when to catalog maps as a single bibliographic record with items attached

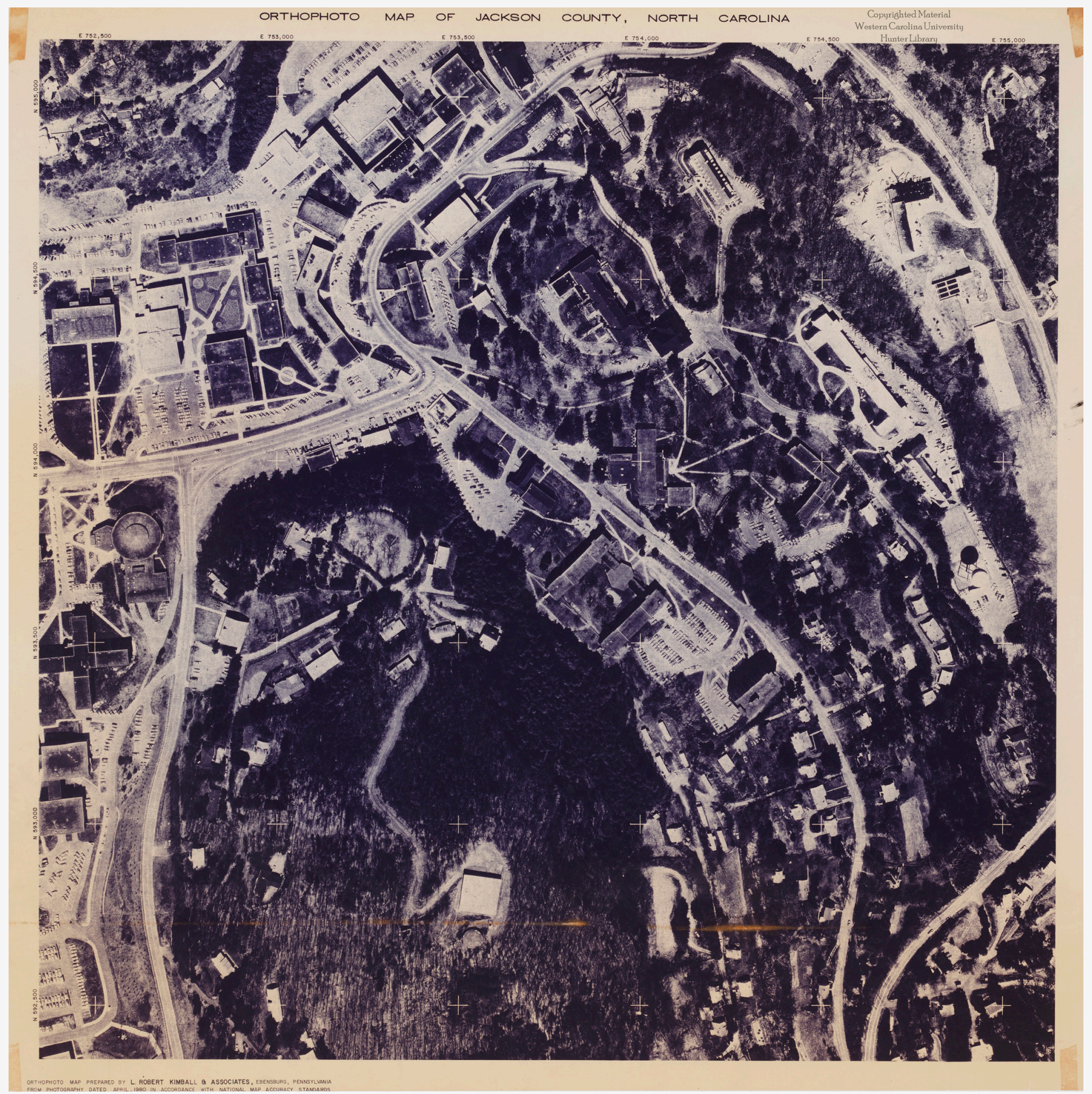

Figure 4. Orthophoto Map of Jackson County, North Carolina, L. Robert Kimball, 1980. 


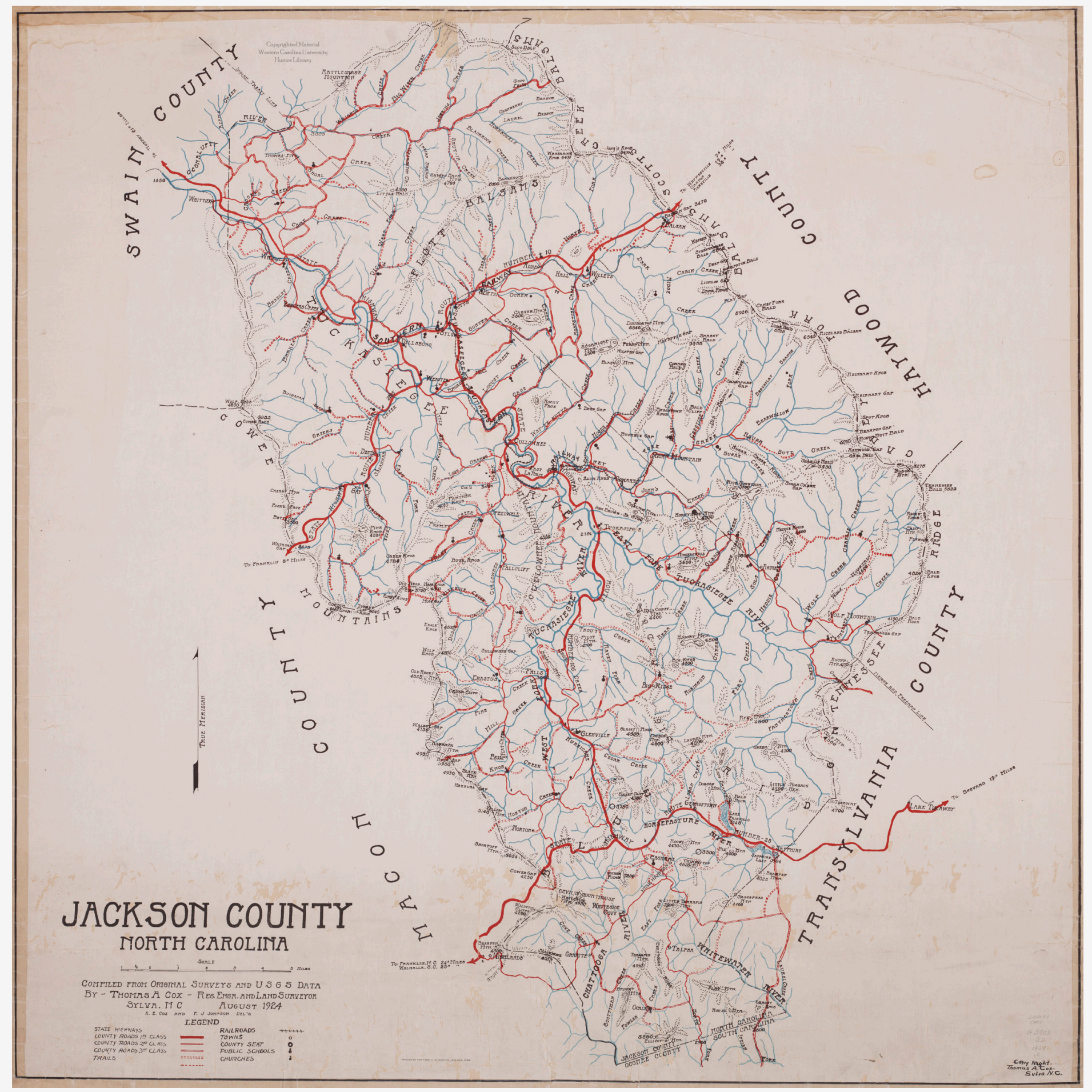

Figure 5. Jackson County, North Carolina, Thomas Cox, 1924.

(one catalog record with multiple maps listed for that record) and when to catalog maps separately. For example, if Jackson County has multiple years of highway maps produced by the state's Department of Transportation, would those maps be cataloged as a single set of Jackson County maps with varying years, or would each be cataloged separately? We generally leaned towards cataloging maps in sets. This option seemed to make the most sense from the perspective of a library patron (related maps are found together) while also minimizing catalog clutter. However, as a trade-off, cataloging in sets meant that individual items received less descriptive detail. 
Determining the appropriate level of description also posed a challenge for individually catalogued maps. The Maps Librarian and the cataloger agreed on what might be too much detail for most maps, such as comprehensive lists of family or place names for particularly detailed maps. They also determined what marks and information were important enough to note, such as the MeigsFreeman Line (surveyed in 1802 as part of the Treaty of Tellico, this demarcation served as a boundary line until 1819 between the United States and the Cherokee).
Once descriptive guidelines were set, we began scanning the maps. As the library has limited in-house resources for scanning very large or fragile materials, the first batch of maps was scanned by the North Carolina Digital Heritage Center at the University of North Carolina at Chapel Hill. Thirty-three maps were sent, representing highlights from both Hunter Library's Special Collections and its general map collection. For maps small enough to be scanned inhouse, we primarily used an overhead Bookeye 4 scanner. Maps were scanned at $600 \mathrm{dpi}$ and saved as TIFF files,

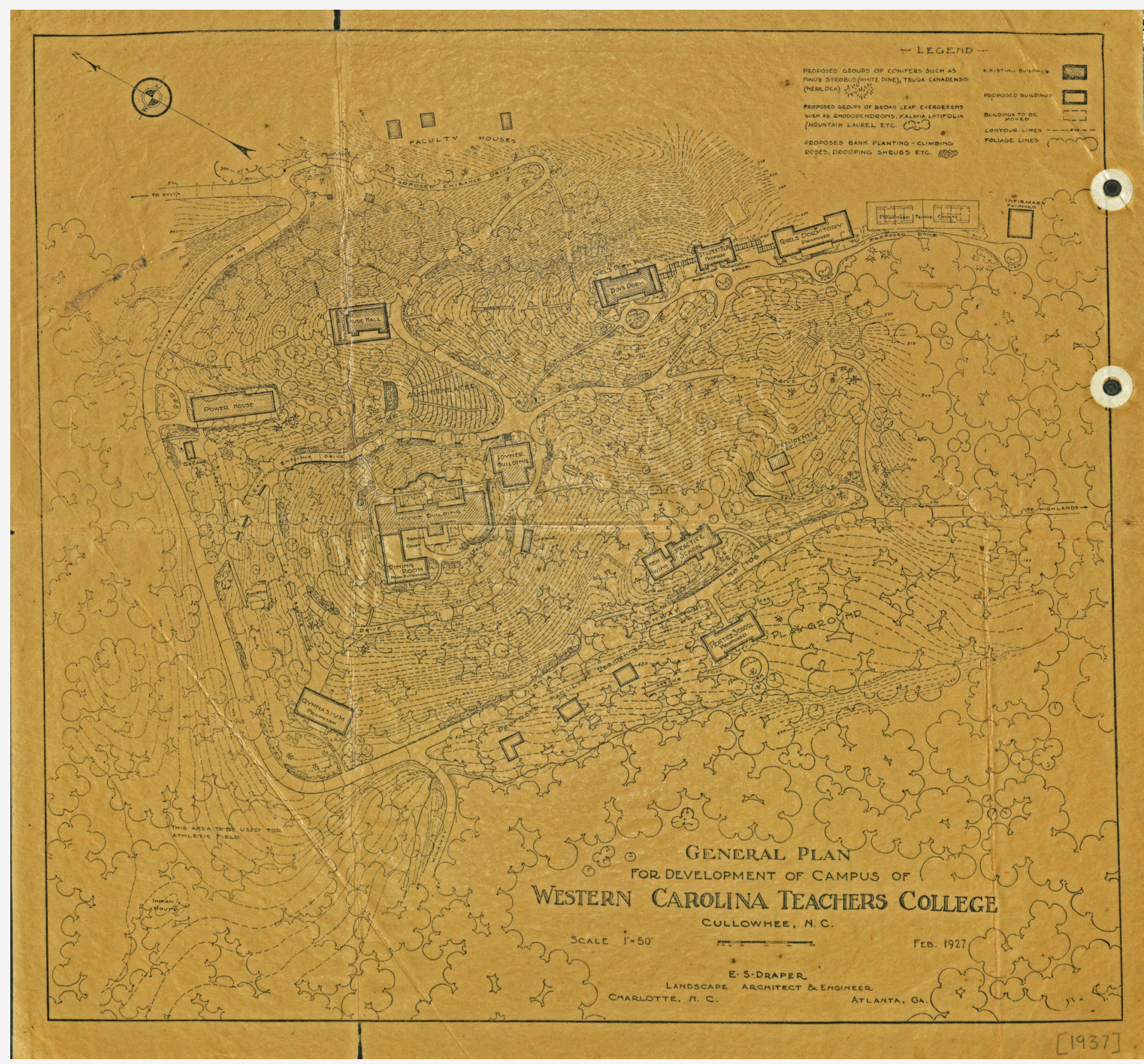

Figure 6. General Plan for Development of Campus of Western Carolina Teachers College, Cullowhee, N.C., E. S. Draper, 1937. 
which captured a high-level of detail, facilitated zooming, and allowed us to print high-quality reproductions. The 23" $\times 34$ " scan bed allowed maps to be laid out and scanned from above, ensuring material safety.

The digital collection debuted four months after the project began, with seventeen of the thirty-three maps chosen for the pilot. To date, there are 130 maps in the collection and those maps have received nearly 4,000 online views. The maps have been used in a variety of student and faculty projects; for example, many maps featured prominently in an upper level history course that focused on some of the region's historic communities that once appeared on maps but no longer do. The 1924 Thomas Cox map of Jackson County served as a foundation for the project and students used it to show where former schools, churches, and post offices once existed.

This project has benefited the library in numerous ways including allowing our patrons to engage more deeply in the region's history and raising awareness of the library's map collections throughout the university and region. As the library looks forward, we intend to develop this collection of unique maps, extend its the scope, and solicit local and regional partners including the town of Lake Lure, with whom discussions are ongoing. In the meantime, we will be adding maps from the 1930s of the Pisgah National Forest, mid-twentieth-century maps from the Blue Ridge Parkway, and more maps of interest from the library's manuscript collections.

A final note to other institutions considering similar projects: we found the most useful steps to be those of the planning process. We defined the scope of our project narrowly enough to be accomplishable, while also being scalable. We also defined the intended audiences for such a collection to help guide selection and prioritization, and determined best methods for describing materials to ensure consistency and aid in browsing and findability. Thanks to this careful planning, the project did not seem overwhelming for any member contributing to the project, yet the work still resulted in a robust and meaningful collection. We are very pleased with these first steps and look forward to building this unique bridge between the university and local communities. 